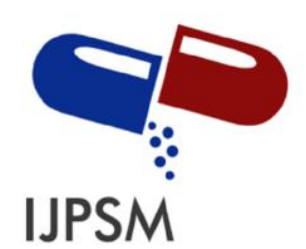

Swapna Singh et al, Int. Journal of Pharmaceutical Sciences and Medicine (IJPSM),

Vol.6 Issue. 1, January- 2021, pg. 1-7

ISSN: 2519-9889

Impact Factor: $\mathbf{3 . 4 2 6}$

\title{
REVIEW: AN APPROACH TO BIOANALYTICAL METHOD DEVELOPMENT AND VALIDATION
}

\author{
Swapna Singh*; Prem Kumar Bichala ${ }^{1}$; Abhishek Agrwal ${ }^{2}$; Ravi Shankar Bhatnagar \\ *School of Pharmaceutical Sciences, Pratap University, Jaipur, Rajasthan, swapnasingh112@ gmail.com \\ ${ }^{1}$ School of Pharmaceutical Sciences, Pratap University, Jaipur, Rajasthan, prem.kumar@ pratapuniversity.in \\ ${ }^{2}$ School of Pharmaceutical Sciences, Pratap University, Jaipur, Rajasthan, abhishek.agrwal@ pratapuniversity.in \\ ${ }^{3}$ R.J. College of Pharmacy, Raipur, Tappal, U.P, rshankarpy002@ gmail.com
}

DOI: 10.47760/ijpsm.2021.v06i01.001

\begin{abstract}
In this review article, a brief discussion of Bioanalytical method development and Validation. The development process to ensure quality and reliability of method development of bio-analytical sample by using Various Validation parameters like accuracy, precision, reproducibility and stability are performed it's limits sets by the FDA (Food and drug Administration).The bioanalytical method includes such as solid phase extraction, Chromatography method etc.

Keywords: Method development, Validation of bioanalysis techniques, Validation parameter.
\end{abstract}

\section{Introduction}

The Bioanalytical method validation is the process used to establish the analytical procedure employed for a specific test is to yield reliable results that can be satisfactorily interpreted.

This bioanalytical method employed for the quantitative determination of drugs and their metabolites in biological fluids. The estimation of bioavailability, bioequivalence, pharmacokinetic, and toxicokinetic study data.These studies generally support regulatory filings for the drug approval from FDA.[1]

\section{Need of Bioanalytical Method Validation}

- The bioanalytical technique has its own characteristics, which will vary from analyte to analyte, specific validation criteria may need to be developed for each analyst.[3]

- The bioanalytical methods to yields to reliable and satisfactorily result interpreted.

- Validation involves documenting, through the utilization of specific laboratory investigations, that the performance characteristics of the technique that are suitable and reliable for the intended analytical applications.[2]. 


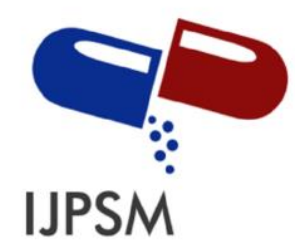

Swapna Singh et al, Int. Journal of Pharmaceutical Sciences and Medicine (IJPSM), Vol.6 Issue. 1, January- 2021, pg. 1-7

ISSN: 2519-9889

Impact Factor: 3.426

\section{Types of Bioanalytical Method Validation}

Bio analytical method validation is classified into three types
A. Full validation
B. Partial validation
C. Cross validation

\section{A. Full Validation:}

The development and implementation in Bio Analytical technique when the new drug molecule has been discovered.

\section{B. Partial Validation:}

The partial validations are the already existing validated bioanalytical methods $\&$ it's modifications. In partial validation doesn't require necessarily to validate full orelse revalidation Partial validation can range from as little as one intra-assay accuracy and precision. bioanalytical method changes that fall into this category includes.

1. Bioanalytical method transfers between laboratories or analysts

2. Change in analytical methodology (e.g., change in detection systems)[4]

3. Change in anticoagulant in harvesting biological fluid

4. Change in sample processing procedures

5. Change in relevant concentration range

6. Changes in instruments and/or software platforms

7. Limited sample volume (e.g., pediatric study)

8. Rare matrices ${ }^{8}$

9. Selectivity demonstration of an analyte in the presence of concomitant medications Selectivity demonstration of an analyte in the presence of specific metabolites[5]

\section{Cross validation:}

The comparison of the validation parameters when two or more bioanalytical methods are wont to generated the info within same study or across different study. (e.g., LC-MS-MS vs. ELISA) in several studies are included during a regulatory submission. 


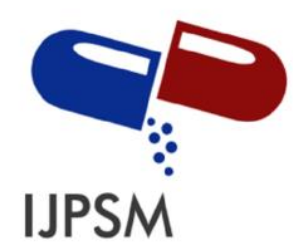

Swapna Singh et al, Int. Journal of Pharmaceutical Sciences and Medicine (IJPSM), Vol.6 Issue. 1, January- 2021, pg. 1-7

ISSN: 2519-9889

Impact Factor: 3.426

\section{Steps Involved in Method Validation:[6]}

1. Development of a validation protocol

2. The Purpose, objectives and plan of the method

3. The performance parameters and acceptance criteria

4. Verify relevant performance characteristics of equipment

5. Qualify materials, for example, standards reagents for purity, accurate amounts, and sufficient stability

6. Perform pre-validation experiments

7. Modifications in method development for Validations

8. Devlop a Standard operating procedures (SOPs)

9. Validate the Bioanalytical sample to ensure quality and reliability and different pharmacokinetics parameters

10. Document validation experiments and results in the validation report.

\section{Bioanalytical method development:}

Liquid-liquid extraction (LLE): This technique is useful for the separating the analytes from interferences by partitioning the sample between two immiscible liquids or phases. i.e., One phase is aqueous while another phase is an organic phase. The polar organic phase is hydrophobic in nature whereas, aqueous phase is an hydrophilic in nature. Analyte extracted into the organic phase are easily recovered by evaporation of the solvent, while analytes extracted in to the aqueous phase can often be injected directly on to a reversed-phase column. This technique is very rapid and efficient as well as less cost Required when compared to the other methods The extraction containing drug can be evaporated to dryness and the residue reconstituted in a smaller volume of an appropriate solvent (preferably mobile phase). Near quantitative recoveries $(90 \%)$ of most drugs can be obtained through multiple continuous extractions.[7,12]

Solid Phase Extraction (SPE): The partitioning is occurring in between liquid and solid phase. This technique is more efficient separation process than LLE. It is easier to obtain a higher recovery of analyte. The major advantage of this method are increases the method sensitivity by removing the impurities present in the analyte (biological sample). In SPE, multiple sampling is possible generally 12-24 very low quantity of solvent with automation are major contributing factors. The SPE consists of the medical syringe which is packed with 0.1 to $0.5 \mathrm{~g}$ of sorbent generally C18 silica. Liquid samples were added to the cartridge and wash solvent is selected to either strongly to get retain or unretain the analyte. To minimize the presence of interferences the analyte is eluted with elution solvent and either directly injected in or evaporated to dryness followed by dilution with the HPLC mobile phase.[13]

Protein Precipitation: It is one of the methods to make the matrix interference free. This can be achieved by the denaturation and precipitation. The widely used precipitating agent such as Trichloroacetic acid and perchloric acid. The Various organic solvents includes such as methanol, 


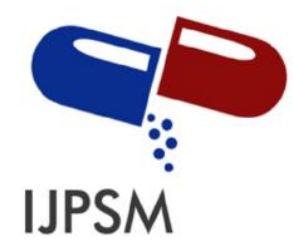

Swapna Singh et al, Int. Journal of Pharmaceutical Sciences and Medicine (IJPSM), Vol.6 Issue. 1, January- 2021, pg. 1-7

ISSN: 2519-9889

Impact Factor: 3.426

acetonitrile, acetone and ethanol are the wide choice for removing plasma proteins and also possess compatibility with high performance liquid chromatography (HPLC) as a mobile phase. One part of sample matrix is diluted with three-four parts of the precipitating agent then mixing is carried out followed by the centrifugation, filtration done for the removal of the protein mass. The supernatant liquid or filtrate is obtained by the directly analyzed for the analyte of interest. For quantitative analysis, the supernatant can be isolated, evaporated to dryness and then reconstituted with a suitable solvent before analysis.[8,9]

Solid Phase Micro extraction (SPME): It is a very simple and solventless method for the extraction, In SPME the fused silica fiber coated with polyacrylate, polydimethylsiloxane, carbowax or other modified bonded phase is kept in contact with the sample and it is exposed to the vapor, also it can be placed in the stream of a gaseous sample to isolate the analyte and concentrate analyte into a range of coating materials. Lastly, the fibers are transferred to analytical instruments like gas chromatography (GC) and GC/mass spectrometry (GC/MS) for separation and quantification of the target analyte with the help of syringe. Exposed fiber has the ability to extract and sample delivery is a key aspect of this method The SPME apparatus is a very simple device. It looks like modified syringe consisting of a fiber holder and a fiber assembly, the latter containing a 1-2 cm long retractable SPME fiber.Analyte in the sample is directly extracted and concentrated to the extraction fiber. The method is advantageous because it's saves the preparation time and disposal costs and can improve detection limits. SPME was also introduced for direct coupling with high-performance liquid chromatography (HPLC) and HPLC-MS in order to analyze weakly volatile.[15]

Matrix Solid-Phase Dispersion (MSPD): In this technique solid matrices are used for sample preparation. The Sample required in a very low quantity with a solvent therefore it is termed as a macroscale extraction technique. Near about $98 \%$ solvent use is reduced and giving $90 \%$ sample turn around time In the Conventional extraction of an organic analyte from tissue, the homogenization of the sample analyte from tissue with bulk bonded silica - based sorbent has to perform, this can be achieved in mortar and pestle. The structure of tissue is getting disturbed due to mechanical shearing. The sample gets dispersed on the surface of support sorbent, for this, hydrophilic and hydrophobic interaction plays a role which causes the mixture to become semi-dry and free-flowing homogeneous blend of the sample. The interferences and analyte are eluted by transferring in to a pre-fitted SPE cartridge. This technique has been applied, by using acid alumina, to extract the organic analyte.[13]

Supercritical Fluid Extraction: This method is generally used for removing the nonpolar to moderately polar analyte from the matrix. The density of the supercritical fluid is like liquid while its viscosity and diffusivity is in the gas and liquid values. By reducing the pressure and by the evaporation, the recovery of supercritical solvent can be obtained. Carbon dioxide dissolves many volatile polar compounds, acting good supercritical solvent. This can be achieved in the presence of trace amounts of 


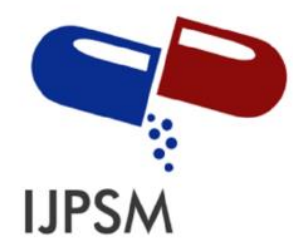

Swapna Singh et al, Int. Journal of Pharmaceutical Sciences and Medicine (IJPSM), Vol.6 Issue. 1, January- 2021, pg. 1-7

ISSN: 2519-9889

Impact Factor: 3.426

polar co-solvents like water and short-chain alcohols. Supercritical fluids can be used to extract analyte from samples.[14]

\section{Validation Parameters:}

Accuracy: The degree of closeness I.e. Nearer value or true value of the observed concentration to the nominal or known true concentration. It is typically measured as relative error (\%RE). Accuracy is an absolute measurement and an accurate method depends on several factors such as specificity and precision.

Accuracy is best reported as percentage bias which is calculated from the expression

$$
\text { Abs\%Bias }=\frac{\text { Measured Value }- \text { True Value }}{\text { True Value }} \times 100
$$

Precision: The precision of a bioanalytical method is a measure of the random error and is defined as the closeness of agreement between a series of measurements obtained by injecting a series of standards or analyzing series of samples from multiple samplings from a homogeneous lot. It is measured by the Coefficient of the Variations.(\%CV)[17]

$$
\% C V=\frac{\text { Standard deviation }}{\text { Mean }} \times 100
$$

Repeatability: It expresses the analytical variabilitv under the same onerating conditions over a short interval of time. It is also known as intra-assav nrecision.( expresses within-laboratories variations: different days, different analysts, different equipment, etc.)

Reproducibility: This expresses the precision between the measurement results obtained at different laboratories. Sometimes a mistake is made and a term reproducibility is used for a within-laboratory studies at the level of intermediate precision.

Range: The interval between the upper concentration of the analyte and lower concentration of the analyte. The range is a bioanalytical assay of the concentration between the intervals over which an analyte can be measured with acceptable precision and accuracy.

Robustness: Robustness can be used to establish system suitability parameters. Robustness is the evaluation of an analytical method wherein the results obtained are found to be reliable even when performed in a slightly varied condition. It is the ability of a method to remain unaffected when slight variations are applied.[10,11] 


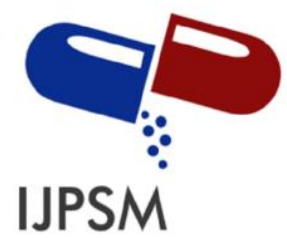

Swapna Singh et al, Int. Journal of Pharmaceutical Sciences and Medicine (IJPSM), Vol.6 Issue. 1, January- 2021, pg. 1-7

ISSN: 2519-9889

Impact Factor: 3.426

Recovery: The extraction efficiency of an analytical process, reported as a percentage of the known amount of an analyte carried through the sample extraction and processing steps of the method [18]. This can be expressed by the following equation.

$$
\text { Absolute recovery }=\frac{\text { Response of analyte spiked into matrix }(\text { processed })}{\text { Response of analyte of pure standard(unprocessed })} \times 100
$$

Stability: The chemical or physical stability of an analyte during a given matrix under specific conditions for given time intervals. The aim of a stability test is to detect any degradation of the analytes The condition under which the stability is determined is largely hooked in to the character of the analyte, the biological matrix, and the anticipated time period of storage (before analysis). The FDA guidelines on bioanalytical method validation also because the recent AAPS/FDA documentation or regulatory filings evaluating analyte stability at different stages. Stability should be confirmed for every step of sample preparation and analysis, as well as the conditions used for long-term storage. Percent stability could be calculated as follows[16]

$$
\% \text { Stability }=\frac{\text { Mean response of stability samples }}{\text { Mean response of comparision samples }} \times 100
$$

\section{Conclusion:}

The bioavailability, bioequivalence, pharmacokinetic, and toxicokinetic study data and their interpreted results can be obtained. This plays a plays a fundamental role in pharmaceutical research and development involved in the drug discovery and development process.

\section{References}

[1]. Kollipara S, Bende G, Agarwal N, Varshney B, Paliwal J (2011) Internatioanl guidelines for Bioanalytical method Validation: A Comparison and Discussion on Current Scenario. Chromatographia 73: 201-217.

[2]. Yadav AK, Singh SK, Yashwant, Verma S (2012) Bioanalytical Method Validation -How, How Much and Why: A Reaseach Perspective. Int J of Nat Prod Sci 1: 123.

[3]. Patil S, Pandurang ND, Kuchekar BS (2009) Bioanalytical method development and validation: Guidelines Latest reviews. pharmainfo.net 7: 1-8.

[4]. Gao L1, Li J, Kasserra C, Song Q, Arjomand A, et al. (2011) Precision and accuracy in the quantitative analysis of biological samples by accelerator mass spectrometry: application in microdose absolute bioavailability studies. Anal Chem 83: 5607-5616 


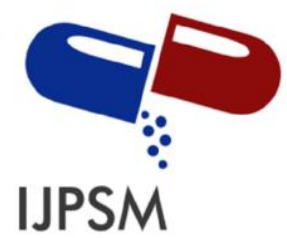

Swapna Singh et al, Int. Journal of Pharmaceutical Sciences and Medicine (IJPSM), Vol.6 Issue. 1, January- 2021, pg. 1-7

ISSN: 2519-9889

Impact Factor: 3.426

[5]. Tiwari G1, Tiwari R (2010) Bioanalytical method validation: An updated review. Pharm Methods 1: 25-38.

[6]. CITAC/EURACHEM, Working Group, International Guide to Quality in Analytical Chemistry:An Aid to Accreditation; 2002.

[7]. Snyder LR, Kirkland JJ, Glajch JL. Practical HPLC Method Development. 2nd ed. USA: John Wiley \& Sons; 2001

[8]. Mark H. Application of improved procedure for testing linearity of analytical method to pharmaceutical analsysis. J Pharm Biomed Anal 2003;33(1):7-20.

[9]. 18. Hartmann C, Smeyers-Verbeke J, Massart DL, McDowall RD. Validation of bioanalytical chromatographic methods. J Pharm Biomed Anal 1998;17(2):193-218.

[10].Dadgar D, Burnett PE. Issues in evaluation of bioanalytical method selectivity and drug stability. J Pharm Biomed Anal 1995;14(1-2):23-31.

[11]. Miller KJ, Bowsher RR, Celniker A, Gibbons J, Gupta S, Lee JW, et al.Workshop on bioanalytical methods validation for macromolecules: Summary report. Pharm Res 2001;18(9):1373-83.

[12]. Causon R. Validation of chromatographic methods in biomedical analysis. Viewpoint and discussion. J Chromatograph B Biomed Sci Appl 1997;689(1):175-80.

[13].S Lakshmana Prabu and T N K Supriyaprakash. Extraction of drug from the biological matrix: a review. Applied biological engineering-Principles and Practice. Available from:http://www.intechopen.com/ooks/applied-biological-engineering-principles-and practice/extraction-of-thedrug-from-the-biological-matrix.

[14].Douglas A Skoog, F James Holler, Timonthy A. Nieman Principles of instrumental Analysis, fifth edition, Thomson Asia Pte Ltd., Singapore., 2003.

[15].Gyorgy Vas and karoly Vekey. Solid-phase microextraction: A powerful sample preparation tool prior to mass spectrometric analysis. Journal of Mass Spectrometry, 39:233-254, 2004.

[16].Hubert H, Chiap P, Crommen J, Boulanger B, Chapuzet E, Mercier N, et al. The SFSTP guide on the validation of chromatographic methods for drug analysis: From the Washington Conference to the laboratory. Anal Chim Acta 1999;391:45-55

[17].Singh PS, Shah G. Analytical method development and validation. J Pharm Res 2011;4(5):2330-2.

[18]. Wood R. How to validate analytical methods. Trends Analyst Chem 2005;18:624-32.

\section{Authors Biography:}

Ms. Swapna Singh, Assistant Professor, School of Pharmaceutical Sciences, Pratap University, Jaipur, Rajasthan, has been awarded the degree of Master of Pharmacy in Pharmaceutical Chemistry from Technocrat institute of technology-Pharmacy, Bhopal. She has 2 years of teaching and research experience.

Mr. Prem Kumar Bichala, Associate Professor, School of Pharmaceutical Sciences, Pratap University, Jaipur, Rajasthan, has been awarded the degree of Master of Pharmacy in Pharmaceutical Analysis by Acharya Nagarjuna University, Guntur, A.P. Presently pursuing Ph.D. from JNT University Kakinada, Kakinada, A.P. He has 10 years of teaching and research experience. He has published various research papers in peer reviewed National \& International journals.

Mr. Abhishek Agrawal, Assistant Professor, School of Pharmaceutical Sciences, Pratap University, Jaipur, Rajasthan, has been awarded the degree of Master of Pharmacy in Pharmaceutical Chemistry from Rajiv Academy for Pharmacy, Mathura. He has 7 years of teaching and research experience.

Ravi Shankar Bhatnagar, R.J. College of Pharmacy, Raipur, Tappal, U.P 